\title{
Chiroptical Symmetry Analysis (CSA): on the Way from the Understanding of Highly Symmetric Systems to the Development of Chiroptical Applications
}

\author{
Silvia Castro-Fernández, ${ }^{1}$ Ángeles Peña-Gallego, ${ }^{2}$ Ricardo A. Mosquera*2 and José Lorenzo \\ Alonso-Gómez ${ }^{* 1}$ \\ 1 Departamento de Química Orgánica, Facultade de Químicas, Universidade de Vigo, Lagoas-Marcosende \\ s/n, Vigo 36310, Spain; lorenzo@uvigo.es \\ 2 Departamento de Química Física, Facultade de Químicas, Universidade de Vigo, Lagoas-Marcosende s/n, \\ Vigo 36310, Spain; mosquera@uvigo.es
}

\begin{abstract}
The high sensitivity of chiroptical responses to conformational changes and supramolecular interactions has prompted an increasing interest in the development of chiroptical applications. However, prediction and understanding the chiroptical responses of the necessary large systems may not be affordable for calculations at high levels of theory. In order to facilitate the development of chiroptical applications, methodologies capable of evaluating the chiroptical responses of large systems are necessary. Exciton chirality method has been extensively used for the interaction between two independent chromophores through the Davydov model. For systems presenting $C_{2}$ or $D_{2}$ symmetry one can get to the same results by applying the selection rules. In the present article, analysis of the selection rules for systems with symmetries $C_{n}$ and $D_{n}$ with $n=3$ and 4 is used to uncover the origin of their chiroptical responses. We hope that the use of the chiroptical symmetry analysis (CSA) for systems presenting the symmetries explored herein as well as for systems presenting higher symmetries will serve as a useful tool for the development of chiroptical applications.
\end{abstract}

Keywords: Chirality; Chiroptical Responses; Exciton Chirality; Selection Rules; Symmetry.

\section{Introduction}

Chirality, or the existence of a pair of non-superimposable mirror-image shapes, is a natural property present in a wide variety of biological systems. While enantiomeric molecules present the same interaction with other achiral molecules, they may interact differently with other chiral molecules or entities. Besides its impact in the biological world is ubiquitous, chirality can also be exploited in other areas such as materials science. For example, the interaction of chiral compounds with light has been used for the construction of light powered molecular motors or in the development of more sensitive and specific sensors. The increasing interest in the potentiality of chiral systems for miscellaneous applications makes essential the understanding of their chiroptical responses and the development of methodologies to study them.

When a linearly polarized light passes through a chiral media, the resulting light can become an elliptically polarized light due to the different absorption of the right and left circularly polarized light by the chiral media[1]. This phenomenon is known as circular dichroism or electronic circular dichroism (CD or ECD). Derived from the particular interaction of chiral molecules with light, optical methodologies were developed to characterize enantiomers according to their absolute configurations[2].

The electronic circular dichroism is one of the most used chiroptical spectroscopies. Comparison of the experimental and theoretically predicted ECD spectra is routinely used not only 
for absolute and relative configuration assignment of small and medium sized molecules[3], but also for elucidating the conformational preferences of the systems under study[4,5]. The level of theory employed for the simulation of the ECD spectra must be carefully chosen according with the nature of the systems under study[6]. As an alternative to the use of ab initio calculations, the exciton chirality method can be applied when two independent chromophores are present in the molecule[7]. The more general phenomenon known as the Davydov splitting [8], 2 $\mathrm{V}_{12}$, merges from the excitation energy splitting between the in-phase and out-of-phase simultaneous two chromophore transitions, strongly dependent on their relative orientation (Scheme 1).

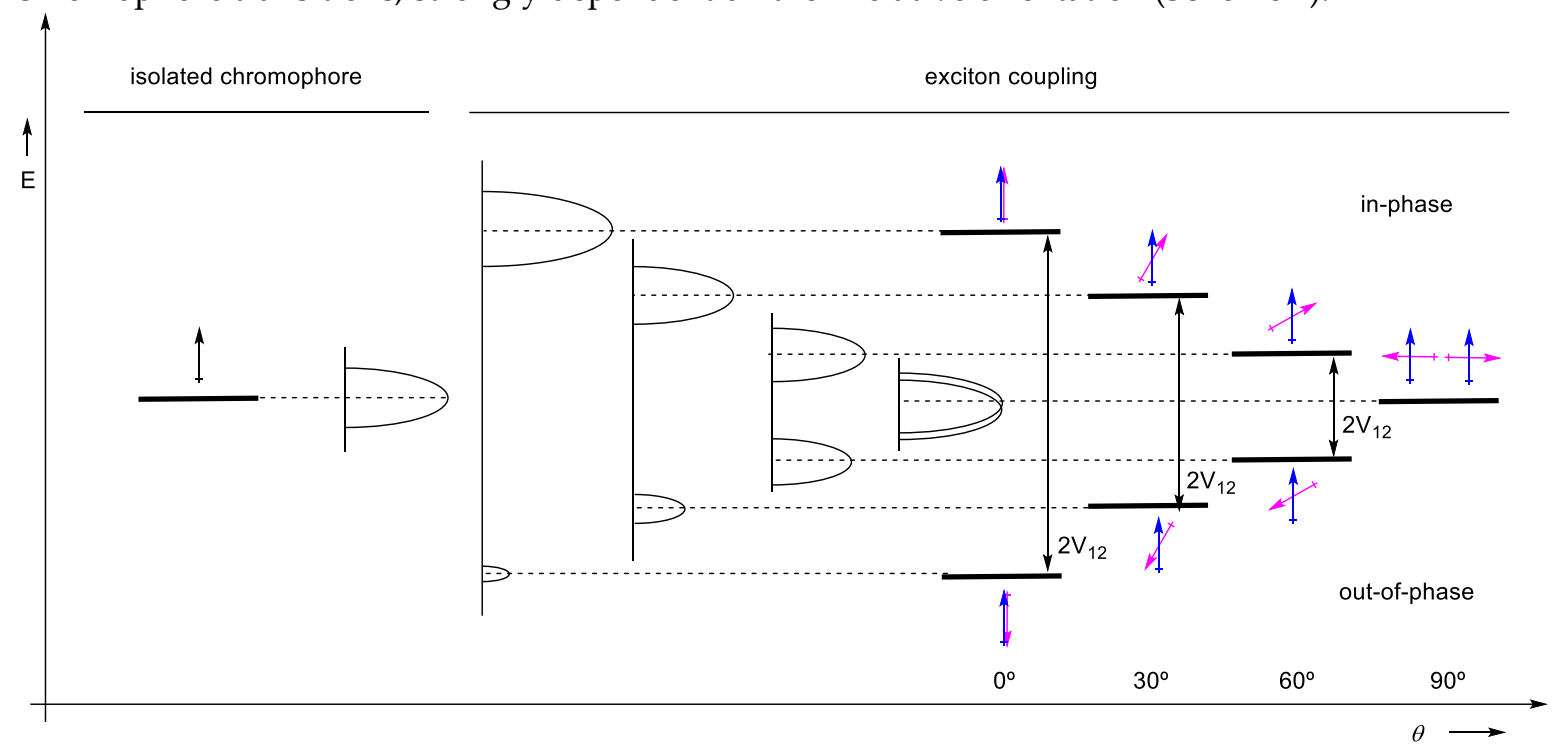

Scheme 1. Representation of the splitting of the energy excitation of two interacting chromophores due to the in-phase and out-of-phase interactions. Arrows represent the electronic transition for two chromophores, blue and magenta. Gaussian curves represent the absorption band in the UV/Vis spectra.

When the arrangement between the interacting chromophores is chiral, the Cotton effects merging from the in-phase and out-of-phase excitations in the CD spectrum present opposite sign. Notable, the sing of the less energetic band in the resulting bisignated CD signal is diagnostic of the handedness of the chiral arrangement between the chromophores allowing the absolute configuration determination of many compounds[7,9] (Scheme 2).

positive exciton coupling

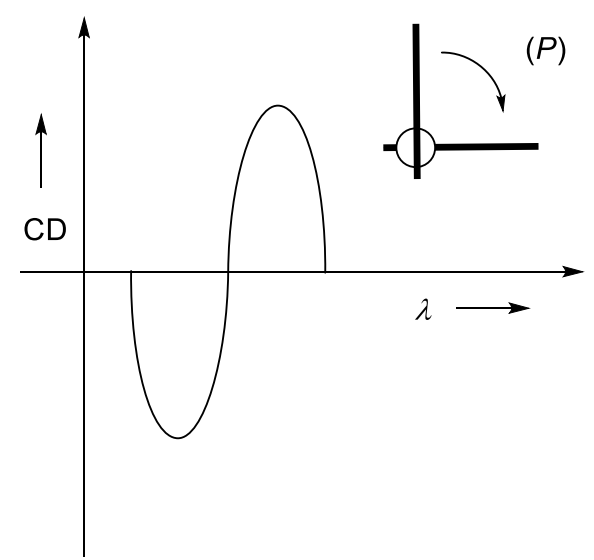

negative exciton coupling

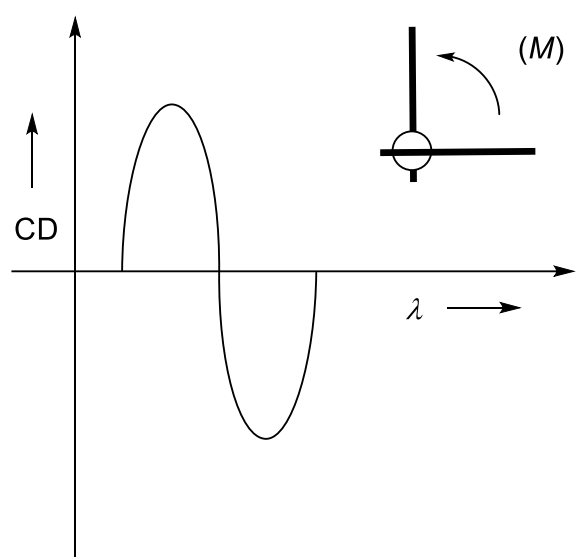

Scheme 2. Representation of bisignated CD signal arising for the interaction between two independent chromophores with a relative positive (left) or negative (right) twist. 
The Davydov model considers an ideal system formed by two non-conjugated chromophores with two-fold degenerated excited states. Perturbation theory allows breaking degeneration considering the interaction between both chromophores. This can be done employing the point dipole approximation. According to it the interaction between two dipoles is given by equation (1). If two chromophores are represented by the corresponding electric transition dipole moment (ETDM), $\mu_{i}$, the interaction between them, $V_{12}$, is given (in au) by equation (1), where $R_{12}$ is the vector connecting the origin of $\mu_{1}$ and $\rho_{2}$. When these chromophores display also permanent dipoles in excited or ground state, represented respectively by, $\mu_{i}$ and $\mu_{i}^{a}$ additional terms have to be considered within $V_{12}$, as it will be shown below.

$$
V_{12}=R_{12}^{-3}\left[\mu_{1}^{t} \cdot \rho_{2}^{t}-3 R_{12}^{-2}\left(\mu_{1}^{t} \cdot \rho_{12}\right)\left(\mu_{2}^{t} \cdot \rho_{12}\right)\right],
$$

The application of the perturbation theory to the (originally degenerated) first electronically excited level yields an energy splitting, $\Delta E$, between these two states (usually named $\alpha$ and $\beta$ ) given by equation (2).

$$
\Delta E=2 V_{12}
$$

This outcome is comparable with the analysis of a $C_{2}$ and $D_{2}$ symmetric systems in terms of the selection rules. In recent year a number of highly symmetric systems such as linear[5] and cyclic oligomers[10], molecular containers[11,12] or even all-carbon double helices[13] presenting remarkable chiroptical responses have been developed on the search for valuable materials for everyday chiroptical applications[14]. However, analysis of the chiroptical responses is often tedious[15,16]. In order to have a more accessible understanding of the behavior of highly symmetric systems, herein we perform the chiroptical symmetry analysis (CSA) that may be applied to systems with $C_{\mathrm{n}}$ or $D_{\mathrm{n}}$, symmetry for $\mathrm{n}=2,3$ or 4 . Additionally, since the CSA is general, it could be applied to any other symmetry, and therefore, we hope that it will serve as a design tool for the development of everyday chiroptical applications in the near future.

\section{Results and discussion}

\subsection{Theoretical background}

The interaction of UV-visible light with a molecule can result in an electric excitation, usually depicted, within the Hartree-Fock framework[17], as the transition from one of the occupied molecular orbitals, $\phi_{i}{ }^{0}$, to an empty (virtual) one, $\phi_{\mathrm{j}}$. This produces a rearrangement of the electron density of the molecule, basically described by the ETDM as a linear displacement of electron density during the transition (Scheme 3). Moreover, the intensity of the transition between the two states is proportional to the square of the modulus of the ETDM, and null values of ETDM correspond to forbidden electronic transitions. The selection rules stablish that for a transition to be allowed at least one of the components of the ETDM must contain the totally symmetric representation of the system point group, and, consequently, the direct product for the representations of the involved wave functions must contain at least one of the representations of the Cartesian coordinates[18].
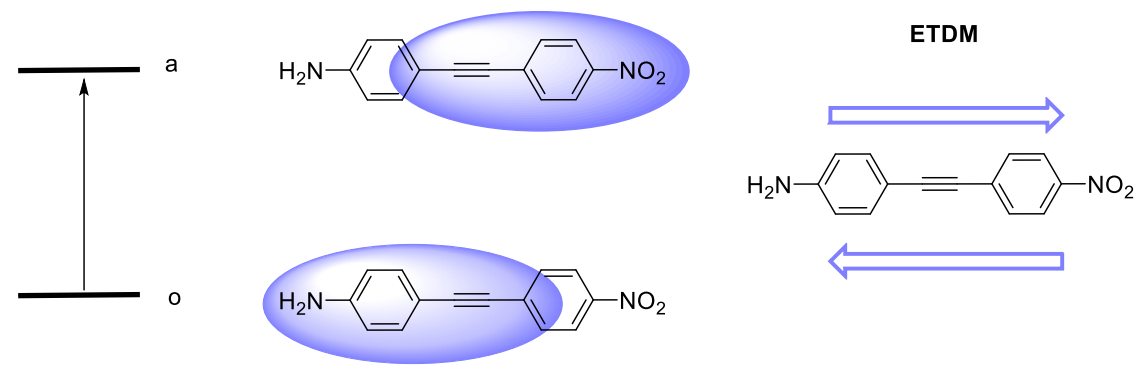
Scheme 3. Representation of the $o \rightarrow a$ electronic excitation of a model compound. The electron density redistribution from the ground state $o$ to the excited state $a$ results in the electric transition dipole moment (ETDM). ETDM has a defined direction and two equally probably orientations.

Davydov's molecular exciton model provides an elegant explanation for the CD Cotton effects in a molecule with two identical chromophores[7]. The model shows how point-dipole interaction between both chromophores splits of the ideally degenerated excited state. Neglecting all kind of interactions between the chromophores (zero-order approximation), the relative energy of the excited state with regard to the ground state would be the same as in an isolated chromophore, $E^{\text {a }}$. In fact, when considering that the electronic transition only affects the chromophores, it is assumed that the rest of the molecule remains unchanged by the transition. Therefore, in the zero-order approach, the ground state, $\Psi^{0(0)}$, can be represented by the product of two ground state wave functions for the isolated chromophore (4), $\psi^{0}$, whereas the zero-order degenerated excited electronic state, $\Psi^{\mathrm{a}(0)}$, can be expressed by the product of ground and excited states, $\psi^{\text {a }}$, of single chromophores, with two equivalent possibilities: $\Psi_{1^{a}(0)}=\psi^{0} \psi^{\text {a }}$ and $\Psi_{2^{a}(0)}=\psi^{\mathrm{a}} \psi^{0}$, where the former refers to the chromophore 1 in the ground state and the chromophore 2 in the excited state.

$$
\Psi^{0(0)}=\psi^{0} \psi^{0},(4)
$$

The application of the perturbation theory to the ground state (non degenerated) provides for the first order correction to the energy, $E^{0(1)}$, the usual expression given by equation (5). Considering only the dipole-dipole interaction between the chromophores, the first-order correction for the energy of the ground state is that shown by equation (6), where $\mu_{i}^{0}$ are the dipole moments of the chromophores in the ground state, named $V_{12}^{00}$.

$$
\begin{array}{r}
E^{0(1)}=\left\langle\psi^{0} \psi^{0}\left|\hat{V}_{12}\right| \psi^{0} \psi^{0}\right\rangle, \\
E^{0(1)}=V_{12}^{00}=R_{12}{ }^{-3}\left[\mu_{1}^{0} \cdot \rho_{2}^{0}-3 R_{12}{ }^{-2}\left(\rho_{1}^{0} \cdot R_{12}\right)\left(\rho_{2}^{0} \cdot R_{12}\right)\right],
\end{array}
$$

Obtaining the first order correction for the energy of the degenerated states leads to a set of linear homogenous equations, with non-trivial solution only when the condition (7) is verified. Ea(1) being the first-order correction to the energy of the excited state for the two-chromophores system. Condition (7) is only fulfilled by the two $E^{a(1)}$ values contained in expression (8). We observe these values involve the dipole-dipole interaction between chromophores in the excited state, $V_{12}^{a a}$, and a term where the interaction applies over the electronic transition, $V_{12}^{t t}$, shown in (9).

$$
\begin{aligned}
& \left|\begin{array}{cc}
\left\langle\psi^{0} \psi^{a} \mid \hat{V}_{12} \psi^{0} \psi^{a}\right\rangle-E^{a(1)} & \left\langle\psi^{0} \psi^{a} \mid \hat{V}_{12} \psi^{a} \psi^{0}\right\rangle \\
\left\langle\psi^{a} \psi^{0} \hat{V}_{12} \psi^{0} \psi^{a}\right\rangle & \left\langle\psi^{a} \psi^{0} \hat{V}_{12} \psi^{a} \psi^{0}\right\rangle-E^{a(1)}
\end{array}\right|=0, \\
& E^{a(1)}=V_{12}^{0 a} \pm V_{12}^{t t}, \\
& \left.V_{12}^{t t}=\left\langle\psi^{0} \psi^{a}\left|\hat{V}_{12}\right| \psi^{a} \psi^{0}\right\rangle=R_{12}{ }^{-3} \mid \mu_{1}^{t} \cdot \rho_{2}^{t}-3 R_{12}{ }^{-2}\left(\mu_{1} \cdot R_{12}\right)\left(\mu_{2} \cdot R_{12}\right)\right] \text {, }
\end{aligned}
$$

Thus, after introducing the first-order corrections obtained with perturbation theory for ground and excited electronic states, the relative energy of the excited state, $\Delta E^{a}$, can be expressed by equation (10). Obviously, the reliability of the formula can be improved including further corrections, but the most significant fact is the splitting of the excited level in two different states (usually named $\alpha$ and $\beta$ ) whose energy differs in $2 V_{12}^{t t}$. Moreover, replacing the two solutions for energy correction (8) in the system of homogeneous equation and normalizing, it is found that the zero-order wave functions for $\alpha$ and $\beta$ states follow, respectively, expressions (11) and (12). 


$$
\begin{aligned}
\Delta E^{a} \cong \Delta E^{a(0)}+\Delta E^{a(1)} & =E^{a}+V_{12}^{0 a}-V_{12}^{00} \pm V_{12}^{t t},(10) \\
\psi_{\alpha}^{0} & =\frac{1}{\sqrt{2}}\left(\psi^{a} \psi^{0}-\psi^{0} \psi^{a}\right),(11) \\
\psi_{\beta}^{0} & =\frac{1}{\sqrt{2}}\left(\psi^{a} \psi^{0}+\psi^{0} \psi^{a}\right),(12)
\end{aligned}
$$

One can directly obtain wave functions (11) and (12) by means of group theory if chromophores are in $C_{2}$ or $D_{2}$ arrangements. Also, their relative energies appear as their averaged $V_{12}$ values (Scheme 4) (13).

$$
\left\langle\psi_{\beta}^{0}\left|\hat{V}_{12}\right| \psi_{\beta}^{0}\right\rangle=\left\langle\psi_{\alpha}^{0} \hat{V}_{12} \psi_{\alpha}^{0}\right\rangle+2 V_{12}^{t t},(13)
$$

$D_{2}$-system

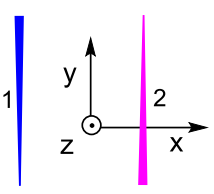

A-transition

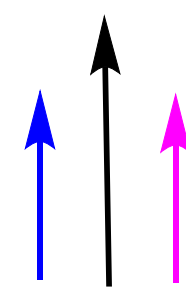

view from $z$

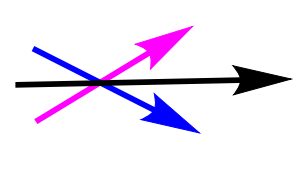

view from $x$
E-transition

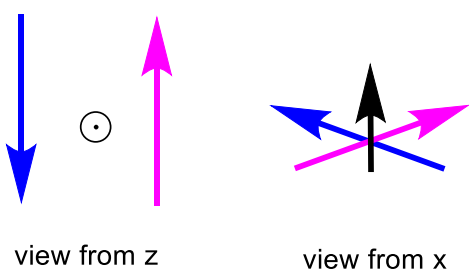

Scheme 4. Representation of a system presenting $D_{2}$ symmetry. According to the selection rules, the two equivalent chromophores 1 (blue) and 2 (magenta) can undergo a simultaneous excitation following A or E symmetry leading to total ETDMs (black) parallel to y or z respectively as a result of the summation of the ETDM of each chromophore. A circle with a dot in the middle represents an arrow perpendicular to the plane pointing to the reader.

\subsection{Excited state splitting with 3 and 4 chromophores}

Let's consider a system formed by three identical chromophores in $C_{3}$ geometry. Assuming that the electronic structure of the three chromophores is basically unchanged with regard to the isolated one, the ground state wave functions could be zero-approached by equation (14) and belong to the totally symmetry irreducible representation of this point group, A. We can expect three equivalent monoexcitations from the ground state, described by the wave functions shown in (15). They can be used as a basis set for constructing $C_{3}$ symmetry-adapted linear combinations (SALC) for first electronically excited states. In this case, the reducible representation decomposes into the symmetry species A and E, as shown in (16). Thus, one A excited state and two E degenerate states can be predicted. They are represented in the zero-approach, respectively, by equations (17) and (18-19). Transitions from the ground state to them are both orbitally allowed.

$$
\begin{array}{r}
\Psi^{0(0)}=\psi^{0} \psi^{0} \psi^{0}, \\
\Psi_{1}^{a(0)}=\psi^{a} \psi^{0} \psi^{0}, \quad \Psi_{2}^{a(0)}=\psi^{0} \psi^{a} \psi^{0}, \quad \Psi_{3}^{a(0)}=\psi^{0} \psi^{0} \psi^{a}, \\
\Gamma\left(\Psi_{1}^{a(0)}, \Psi_{2}^{a(0)}, \Psi_{3}^{a(0)}\right)=A \oplus E,
\end{array}
$$




$$
\begin{array}{r}
\Psi_{A}^{a(0)}=\frac{1}{\sqrt{3}}\left[\psi^{a} \psi^{0} \psi^{0}+\psi^{0} \psi^{a} \psi^{0}+\psi^{0} \psi^{0} \psi^{a}\right], \\
\Psi_{E 1}^{a(0)}=\frac{1}{\sqrt{6}}\left[2 \psi^{a} \psi^{0} \psi^{0}-\psi^{0} \psi^{a} \psi^{0}-\psi^{0} \psi^{0} \psi^{a}\right], \\
\Psi_{E 2}^{a(0)}=\frac{1}{\sqrt{2}}\left[\psi^{0} \psi^{a} \psi^{0}-\psi^{0} \psi^{0} \psi^{a}\right],
\end{array}
$$

\section{$D_{3}$-system}

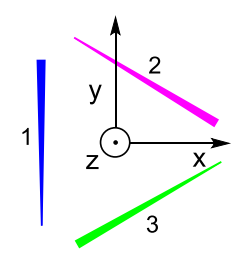

$\mathrm{A}_{2}$-transition

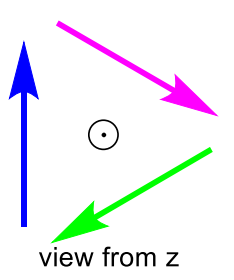

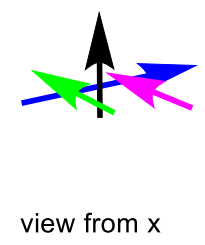

E-transition
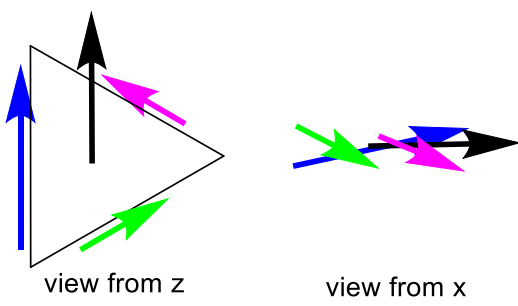

Scheme 5. Representation of a system presenting $D_{3}$ symmetry. According to the selection rules, the three equivalent chromophores 1 (blue), 2 (magenta), and 3 (green) can undergo a simultaneous excitation following A or E symmetry leading to total ETDMs (black) parallel to $\mathrm{z}$ or y respectively as a result of the summation of the ETDM of each chromophore. A circle with a dot in the middle represents an arrow perpendicular to the plane pointing to the reader.

Considering the interaction between the three chromophores is pair additive (20), and the interactions between all pairs are equal, the first-order correction for the energies for the ground, A and $\mathrm{E}$ states are shown in (21)-(23). So, in this case, the excited level splitting is $3 V_{12}^{t t}$, and therefore can be also calculated by means of Davydov's equation.

$$
\begin{array}{r}
\hat{V}=\hat{V}_{12}+\hat{V}_{13}+\hat{V}_{23}, \\
E^{0(1)}=3 V_{12}^{00}, \\
E_{A}^{a(1)}=2 V_{12}^{a 0}+V_{12}^{00}+2 V_{12}^{t t}, \\
E_{E}^{a(1)}=2 V_{12}^{a 0}+V_{12}^{00}-V_{12}^{t t},
\end{array}
$$

The situation in a $D_{3}$ arrangement does not change significantly (nomenclature for the totally symmetry species changes from $A$ to $A_{2}$ ) and the splitting between excited $E$ and $A_{1}$ states is also given by $3 V_{12}^{t t}$.

Considering a $C_{4}$ structure including 4 chromophores, a pairwise approach for the interaction among them, where $\mathrm{V}_{12}$ and $\mathrm{V}_{13}$ are not equivalent, (24) the same approximations, and a similar treatment to that shown above, leads to the following conclusions: i) There are four monoexcited states, two of them degenerate, belonging to the $\mathrm{E}$ symmetry species, the other with $\mathrm{A}$ and $\mathrm{B}$ symmetry; ii) The electronic transition from the ground state to the B one is orbitally forbidden, whereas those to A and E are allowed; iii) SALC for A species (25) represents the zero order approach to the wave function for this state, whereas any linear combination of the two E SALC ((26) and (27)) is possible; and iv) The energy of state A differs from that of $\mathrm{E}$ in $2 V_{12}^{t t}+2 V_{13}^{t t}$; which is the 
splitting in this case. As in the 3-chromophores case, the particularization from $C_{n}$ to $D_{n}$ symmetry has only effects on the nomenclature (A turns into $\mathrm{A}_{2}$ ).

$D_{4}$-system

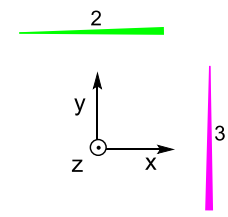

4

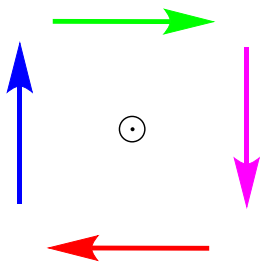

view from $z$
$A_{2}$-transition

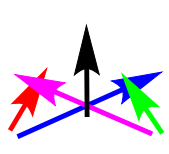

view from $x$
E-transition

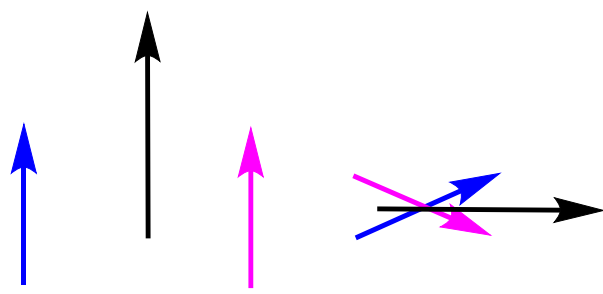

view from $z$

view from $x$

Scheme 6. Representation of a system presenting $D_{4}$ symmetry. According to the selection rules, the four equivalent chromophores 1 (blue), 2 (magenta), 3 (green), and 4 (red) can undergo a simultaneous excitation following A or E symmetry leading to total ETDMs (black) parallel to z or y respectively as a result of the summation of the ETDM of each chromophore. A circle with a dot in the middle represents an arrow perpendicular to the plane pointing to the reader.

$$
\begin{aligned}
& \hat{V}=4 \hat{V}_{12}+2 \hat{V}_{13} \\
& \Psi_{A}^{a(0)}=\frac{1}{2}\left[\psi^{a} \psi^{0} \psi^{0} \psi^{0}+\psi^{0} \psi^{a} \psi^{0} \psi^{0}+\psi^{0} \psi^{0} \psi^{a} \psi^{0}+\psi^{0} \psi^{0} \psi^{0} \psi^{a}\right] \\
& \Psi_{E, 1}^{a(0)}=\frac{1}{\sqrt{2}}\left[\psi^{a} \psi^{0} \psi^{0} \psi^{0}-\psi^{0} \psi^{0} \psi^{a} \psi^{0}\right] \\
& \Psi_{E, 2}^{a(0)}=\frac{1}{\sqrt{2}}\left[\psi^{0} \psi^{a} \psi^{0} \psi^{0}-\psi^{0} \psi^{0} \psi^{0} \psi^{a}\right]
\end{aligned}
$$

\subsection{Rotatory strength}

According to Rosenfeld's quantum theory,[19] the rotatory strength, $R$, defined as the difference between the absorption rate of left circular polarized light (LCP) and right circular polarized light (RCP) in a certain transition, is given by (28), where $\mu_{e}$ and $\mu_{m}$ are, respectively the operators for electric (29) and magnetic dipole moment (30). Both are summation of one-electron operators. Within the context of the independent exciton model the summation only involves the $N$ electrons provided by the chromophores and these electrons can be assigned to a certain chromophore $j$. Moreover, the ratio between $R$ and the integrated absorption coefficient, $A$, (experimentally measurable) is a product of constants, $C,(31)$.

$$
\begin{array}{r}
R=\operatorname{Im} \mid\left\langle\Psi^{0} \mid \hat{\mu}_{e} \Psi^{a}\right\rangle \cdot\left\langle\Psi^{a}\left|\hat{\mu}_{m}\right| \Psi^{0}\right\rangle, \\
\mu_{e}=e \sum_{j} \sum_{k} \rho_{j k}, \\
\mu_{e}=\frac{e \eta}{2 m c i} \sum_{j} \sum_{k} \rho_{j k} \times \nabla_{j k}=\frac{e}{2 m c} \sum_{j} \sum_{k} \rho_{j k} \times P_{j k},
\end{array}
$$




$$
A=\int \frac{\varepsilon(v)}{v} d v=C R
$$

Rosenfeld's equation states that $R$ depends on the scalar product of the ETDM and the corresponding magnetic transition dipole moment (MTDM) as it will be described below. Consequently, optical activity in a certain transition implies: i) transition is both electric dipole and magnetic dipole allowed; and ii) ETDM and MTDM for the process are not mutually orthogonal.

\subsubsection{Electric transition dipole moment}

The fact that ETDM are origin independent and the form of equation (29) allows defining an ETDM vector for each chromophore, $\mu_{j}$ (32). The molecular ETDM results a specific combination of individual vectors for each state $(\alpha$ or $\beta$ ). For example, in the binary system, ETDM follows expression (33) in the $\alpha$ state and expression (34) in the $\beta$ one. The square modulus of the molecular ETDM (directly related to Einstein coefficients in ETDM transitions) depends, in both cases, on the angle between these vectors, $\gamma$. This, in the case of two identical chromophores, expressions (35) and (36) are respectively followed for transitions to $\alpha$ and $\beta$ states. Let us now think about the $\gamma$ angle. For two isolated identical chromophores, the three components of their vectors will be equal. The only difference in the independent exciton model is the chromophores are placed in specific positions in the molecule, introducing different orientation for the groups. The $\gamma$ angle is the one measuring this relative orientation. As a consequence, when the two chromophores are not orthogonally arranged in the molecule the absorptions for $0 \rightarrow \alpha$ and $0 \rightarrow \beta$ transitions are of different intensity.

$$
\begin{array}{r}
\rho_{j}=e \sum_{k}\left\langle\psi^{0}\left(\rho_{j k}\right) \rho_{j k} \psi^{a}\left(\rho_{j k}\right)\right\rangle, \\
\rho_{t \alpha}=\frac{\rho_{1}-\rho_{2}}{\sqrt{2}}, \\
\rho_{t \beta}=\frac{\rho_{1}^{t}+\rho_{2}^{t}}{\sqrt{2}}, \\
\left|\rho_{t \alpha}\right|^{2}=\left|\rho_{t}\right|^{2}(1-\cos \gamma), \\
\left|\rho_{t \beta}\right|^{2}=\left|\rho_{t}\right|^{2}(1+\cos \gamma),
\end{array}
$$

Extending this procedure to $C_{3}$ systems, the expressions (37) and (38) are obtained for molecular ETDM from ground to E and A electronically excited states, and (39) and (40) for the corresponding squared modulus.

$$
\begin{array}{r}
\rho_{t E}=\frac{\rho_{1}^{t}-\rho_{2}}{\sqrt{2}}, \\
\rho_{t A}=\frac{\rho_{1}+\rho_{2}+\rho_{3}}{\sqrt{3}}, \\
\left|\rho_{t E}\right|^{2}=\left|\rho_{1}\right|^{2}(1-\cos \gamma),
\end{array}
$$




$$
\left|\rho_{t A}\right|^{2}=\left|\mu_{t}\right|^{2}(1+2 \cos \gamma)
$$

Similarly, the expressions for molecular ETDM in $C_{4}$ systems ((41) and (42)) show that the absorption for $0 \rightarrow \mathrm{A}$ and $0 \rightarrow \mathrm{E}$ transitions depends on the molecular geometry. In this case the angles between $\rho_{c}$ vectors introverted by $\hat{C}^{4}$ and $\hat{C}^{2}$ operations are respectively represented by $\gamma_{12}$ and $\gamma_{13}$ in equations (43) and (44).

$$
\begin{array}{r}
\rho_{t E}=\frac{\rho_{1}-\rho_{3}}{\sqrt{2}}, \\
\rho_{t A}=\frac{\rho_{t}+\rho_{t}+\rho_{3}+\rho_{4}}{2} \\
\left|\rho_{t E}\right|^{2}=\left|\rho_{1}\right|^{2}\left(1-\cos \gamma_{13}\right), \\
\left|\rho_{t A}\right|^{2}=\left|\rho_{t}\right|^{2}\left(1+2 \cos \gamma_{12}+\cos \gamma_{13}\right)
\end{array}
$$

\subsubsection{Magnetic transition dipole moment}

Within the hypothesis of the independent exciton model, the molecular MTDM for a certain transition $(t: 0 \rightarrow a)$ contained in Rosenfeld's expression can be obtained as the vector summation of group MTDM defined for each chromophore, $j$. Every one of these group MTDM is represented by a vector, $\stackrel{M}{t}_{j}$, defined by expression (45), where subscript $k$ refers to the electrons in chromophore $j$.

$$
\stackrel{\rho}{t}_{j}^{t}=\frac{e}{2 m c} \sum_{k}\left\langle\psi^{0}\left(\rho_{j k}\right) \rho_{j k} \times \rho_{j k} \mid \psi^{a}\left(\rho_{j k}\right)\right\rangle
$$

${\stackrel{\omega}{M_{j}}}_{j}$ vectors are origin-dependent. Thus, their summation should be done considering a common origin for all the electron position vectors, $P_{j k}$. Introducing a reference point for each chromophore (e.g. its center of mass), with position vector $\breve{R}_{j}$, and the relative positions for the electrons in their chromophore, $\stackrel{P}{j k}_{j}^{\prime},(45)$ turns into (46), where $\stackrel{\omega}{M}_{j}^{t}$ splits into two parts.

$$
\stackrel{\rho}{t}_{j}^{t}=\frac{e}{2 m c}\left[\rho_{j} \times \sum_{k}\left\langle\psi^{0}\left(\rho_{j k}\right) \rho_{j k} \psi^{a}\left(\rho_{j k}\right)\right\rangle\right]+\frac{e}{2 m c} \sum_{k}\left\langle\psi^{0}\left(\rho_{j k}\right) \rho_{j k} \times \rho_{j k} \mid \psi^{a}\left(\rho_{j k}\right)\right\rangle,
$$

The first addend contains de linear transition moment of the chromophore, $\rho_{j}^{t}$, defined by (47), whereas the second one defines the MTDM of the isolated chromophore, $\varphi_{j}{ }_{j}$, (48). This allows to relate (equation (49)) the MTDM of the chromophore in the molecule, $\mu_{j}^{t}$, with its value in an isolated species, $\rho_{j}$.

$$
\rho_{j}^{t}=\sum_{k}\left\langle\psi^{0}\left(\rho_{j k}\right) \rho_{j k} \psi^{a}\left(\rho_{j k}\right)\right\rangle
$$




$$
\begin{array}{r}
P_{j}^{t}=\frac{e}{2 m c} \sum_{k}\left\langle\psi^{0}\left(\rho_{j k}\right) \rho_{j k}^{t} \times \rho_{j k} \psi^{a}\left(\rho_{j k}\right)\right\rangle, \\
\rho_{j}^{t}=\frac{e\left(\widetilde{R}_{j} \times \rho_{j}^{t}\right)}{2 m c}+m_{j}^{t},
\end{array}
$$

Simple relations of operators algebra outlined below (equations (50) to (52)) allow to replace $\rho_{j}^{t}$ by the ETDM of the isolated chromophore, $\mu_{j}^{t}$, leading to equation (53), where $v^{t}$ is the wave number for the transition.

$$
\begin{aligned}
& \left\langle\psi^{0}\left[\hat{H}, \rho_{k}\right] \psi^{a}\right\rangle=\left\langle\psi^{0} \mid \hat{H} P_{k} \psi^{a}\right\rangle-\left\langle\psi^{0} P_{k} \mid \hat{H} \psi^{a}\right\rangle= \\
& =\left\langle P_{k} \psi^{a}|\hat{H}| \psi^{0}\right\rangle-\left\langle\psi^{0}\left|P_{k}\right| \hat{H} \psi^{a}\right\rangle=\left(E^{0}-E^{a}\right)\left\langle\psi^{0} P_{k} \psi^{a}\right\rangle=\frac{\left(E^{0}-E^{a}\right) \rho_{t},}{e} \\
& \left\langle\psi^{0}\left[\hat{H}, \rho_{k}\right] \psi^{a}\right\rangle=\left\langle\psi^{0}\left[\left(\frac{\hat{p}_{k}^{2}}{2 m}+\hat{V}\right), \rho_{k}\right] \psi^{a}\right\rangle=\frac{1}{2 m}\left\langle\psi^{0}\left[\hat{p}_{k} \hat{p}_{k}, \rho_{k}\right] \psi^{a}\right\rangle= \\
& =\frac{1}{2 m}\left\langle\psi^{0} \hat{p}_{k} \hat{p}_{k} \rho_{k}-\rho_{k} \hat{p}_{k} \hat{p}_{k}+\hat{p}_{k} \rho_{k} \hat{p}_{k}-\hat{p}_{k} \rho_{k} \hat{p}_{k} \mid \psi^{a}\right\rangle=\frac{1}{2 m}\left\langle\psi^{0} \mid \hat{p}_{k}\left[\hat{p}_{k}, \rho_{k}\right]-\left[\rho_{k}, \hat{p}_{k}\right] \hat{p}_{k} \psi^{a}\right\rangle=\frac{\eta}{m i} \underset{p}{p} \\
& \stackrel{\bar{p}}{p}=\frac{m i\left(E^{0}-E^{a}\right)}{e \eta} \rho_{t}=\frac{2 \pi m c i v^{t}}{e} \rho_{t} \\
& \mu_{j}^{t}=i \pi v^{t}\left(\breve{R}_{j} \times \varpi_{j}\right)+\underline{h}_{j}
\end{aligned}
$$

At this point, summation of $\ddot{M}_{j}^{t}$ vectors leads to molecular MTDM. This is shown for a binary system in states $\alpha$ and $\beta$ in equations (54) and (55), for a $C_{3}$ one in states $E$ and $A$ in equations (56) and (57), and for $C_{4}$ in $\mathrm{E}$ and $\mathrm{A}$ states in (58) and (59).

$$
\begin{aligned}
& \stackrel{\rho}{ }^{t \alpha}=\frac{1}{\sqrt{2}}\left[i \pi v^{t}\left(\rho_{1} \times \widetilde{\mu}_{1}-\rho_{2} \times \varpi_{2}\right)+\left(\rho_{1}-r \rho_{2}\right)\right], \\
& \stackrel{\rho}{t \beta}^{t \beta}=\frac{1}{\sqrt{2}}\left[i \pi v^{t}\left(R_{1} \times \overline{\mu_{1}}+\rho_{2} \times \overline{\mu_{2}}\right)+\left(\rho_{1}^{t}+m_{2}\right)\right]
\end{aligned}
$$

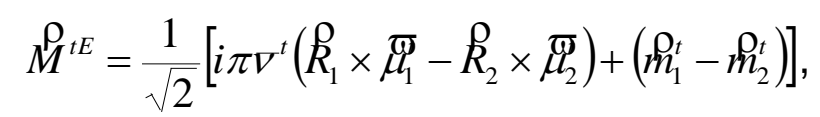

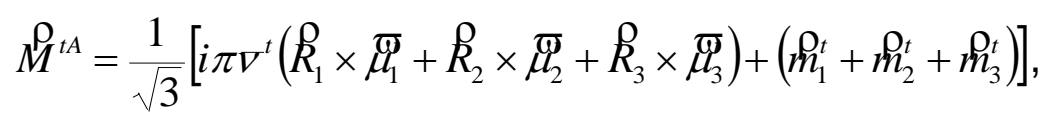

$$
\begin{aligned}
& \stackrel{\rho}{ }^{t E}=\frac{1}{\sqrt{2}}\left[i \pi v^{t}\left(R_{1} \times \varpi_{1}-R_{3} \times \varpi_{3}\right)+\left(m_{1}-m_{3}\right)\right]
\end{aligned}
$$




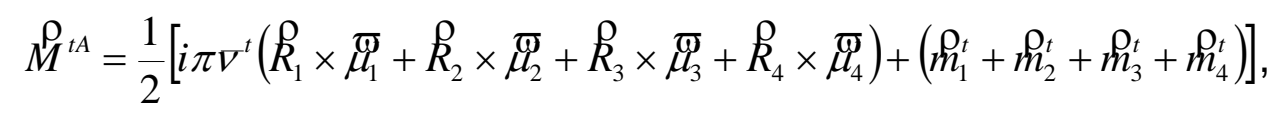

\subsubsection{Rotatory strength in $\mathrm{C}_{n}$ systems $(2 \leq \mathrm{n} \leq 4)$}

The application of Rosenfeld's equation in a binary system to excitations with negligible MTDM for isolated chromophores leads to conclude that rotatory strengths for transitions to $\alpha$ and $\beta$ states are equivalent but with opposite signs (60). In a general case the component due to MTDM are not null. Therefore, the absolute value of the rotatory strength is different for $\alpha$ (61) and $\beta$ states (62).

$$
\begin{array}{r}
R^{\alpha}=-R^{\beta}=\frac{\pi v^{t}}{2}\left(R_{2}-R_{1}\right) \cdot\left(\rho_{1} \times \rho_{2}\right), \\
R^{\alpha}=\frac{1}{2} \operatorname{Im}\left\{\left(\mu_{1}-\rho_{2}\right) \cdot\left(m_{1}-\rho_{2}\right)\right\}+\frac{\pi v^{t}}{2}\left(\rho_{2}-R_{1}\right) \cdot\left(\rho_{1} \times \rho_{2}\right)=R^{E}\left(C_{3}\right), \\
R^{\beta}=\frac{1}{2} \operatorname{Im}\left\{\left(\mu_{1}+\rho_{t}\right) \cdot\left(\rho_{t}+m_{2}\right)\right\}-\frac{\pi v^{t}}{2}\left(R_{2}-\rho_{1}\right) \cdot\left(\rho_{1} \times \rho_{2}\right),
\end{array}
$$

Expression (61) is also valid for the transitions to E states in a $C_{3}$ system. In fact, the expression for ETDM and MTDM in one of the E SALC are equivalent to those of the $\alpha$ state in a binary system. In contrast, the rotatory strength for the transition to the A state follows expression (63), which generalizes all transitions to A states in $C_{n}$ systems.

$$
\begin{aligned}
& R^{A}=\frac{1}{3} \operatorname{Im}\left\{\left(\mu_{1}+\rho_{2}+\rho_{3}\right) \cdot\left(\rho_{t}+m_{2}+m_{3}\right)\right\}+ \\
& -\frac{\pi v^{t}}{3}\left[\left(R_{2}-R_{1}\right) \cdot\left(\mu_{1} \times \rho_{2}\right)+\left(R_{3}-\rho_{2}\right) \cdot\left(\rho_{2} \times \rho_{3}\right)+\left(R_{3}-R_{1}\right) \cdot\left(\rho_{1} \times \rho_{3}\right)\right]
\end{aligned}
$$

Finally, the rotatory strength for $C_{4}$ systems for both allowed transitions follow specific expressions: (64) for E states, where ETDM and MTDM involved correspond to chromophores in 1,3-arrangements, and (65) for the A state.

$$
\begin{aligned}
& R^{E}\left(C_{4}\right)=\frac{1}{2} \operatorname{Im}\left\{\left(\mu_{1}-\rho_{3}\right) \cdot\left(\rho_{1}-m_{3}\right)\right\}+\frac{\pi v^{t}}{2}\left(R_{3}-R_{1}\right) \cdot\left(\rho_{1} \times \rho_{3}\right), \\
& R^{A}=\frac{1}{n} \operatorname{Im}\left\{\left(\sum_{i=1}^{n} \rho_{i}\right) \cdot\left(\sum_{i=1}^{n} \rho_{1}\right)\right\}-\frac{\pi \bar{v}^{t}}{n}\left[\sum_{i=1}^{n-1} \sum_{j=i+1}^{n}\left(\rho_{j}-\rho_{i}\right) \cdot\left(\rho_{i} \times \rho_{j}\right)\right],
\end{aligned}
$$

Alternatively, Since the total ETDM and MTDM can be calculated as discussed above, the rotatory strength for each allowed transition, $R^{I}$, can be directly calculated from the dot product between both total transition dipole moments associated to such transition (66):

$$
R^{I}=\operatorname{Im}\left\{\underline{\mu}^{t I} \cdot \ddot{M}^{t I}\right\}
$$




\section{Conclusions}

It has been shown how the symmetry chiroptical analysis (SCA) may significantly contribute in uncovering the chiroptical responses of highly symmetric systems bearing independent chromophores given that: i) $V_{i j}$ can be calculated according to Davydov equation; ii) the allow transitions can be predicted by applying the selection rules; iii) $\Delta E$ between those transitions can be calculated according to theory; iv) the symmetry of the allowed transitions enables simple calculation of the total ETDM, MTDM, and rotatory strength associated.

This approach does not intent to accurately predict the experimental $C D$ response of a particular system, but rather to be employed as a useful tool for the design of valuable systems for chiroptical applications. Next, we intend to develop a software for a straight forward analysis of diverse systems to explore the scope of the symmetry chiroptical analysis (SCA).

Author Contributions: Analysis of the selection rules for the different symmetries, S.C.F.; Contextualization of the Symmetry Chiroptical Analysis within the state-of-the-art, A.P.G.; Theoretical demonstration, R.A.M.C; Conceptualization, J.L.A.G.

Funding: This research was funded by Spanish Government grant number CTQ2014-58629-R, RYC-2012-10364, and CTQ2013-50575-EXP" and Galician Government, EM2013/017, GRC2015/17, and ED431F 2016/005.

Acknowledgments: N. Berova (Columbia University, New York, USA), and F. Santoro (ICCOM-CNR-Pisa, Italy) are acknowledged for fruitful discussions.

Conflicts of Interest: The authors declare no conflict of interest. The founding sponsors had no role in the design of the study; in the collection, analyses, or interpretation of data; in the writing of the manuscript, and in the decision to publish the results. 


\section{References}

1. Barron, L. D. Molecular Light Scattering And Optical Activity; Cambridge University press: New York, 2004;

2. Pescitelli, G.; Di Bari, L.; Berova, N.; El-Hachemi, Z.; Naubron, J.-V.; Balaban, T. S.; Bussotti, L.; Atsbeha, T.; Marcelli, A.; Foggi, P.; Berti, D.; Mannini, M. Application of electronic circular dichroism in the study of supramolecular systems. Chem. Soc. Rev. 2014, 43, 5211-5233, doi:10.1039/C4CS00104D.

3. Petrovic, A. G.; Navarro-Vázquez, A.; Alonso-Gómez, J. L. From Relative to Absolute Configuration of Complex Natural Products: Interplay Between NMR, ECD , VCD , and ORD Assisted by ab initio Calculations. Curr. Org. Chem. 2010, 1612-1628.

4. Alonso-Gómez, J. L.; Petrovic, A. G.; Harada, N.; Rivera-Fuentes, P.; Berova, N.; Diederich, F. Chiral induction from allenes into twisted 1,1,4,4-tetracyanobuta-1,3-dienes (TCBDs): conformational assignment by circular dichroism spectroscopy. Chem. Eur. J. 2009, 15, 8396-8400, doi:10.1002/chem.200900103.

5. Rivera-Fuentes, P.; Alonso-Gómez, J. L.; Petrovic, A. G.; Santoro, F.; Harada, N.; Berova, N.; Diederich, F. Amplification of chirality in monodisperse, enantiopure alleno-acetylenic oligomers. Angew. Chem. Int. Ed. 2010, 49, 2247-2250, doi:10.1002/anie.200906191.

6. Petrovic, A. G.; Berova, N.; Alonso-Gómez, J. L. Structure Elucidation in Organic Chemistry. In Structure Elucidation in Organic Chemistry: The Search for the Right Tools; Cid, M.-M., Bravo, J., Eds.; Wiley-VCH Verlag GmbH \& Co. KGaA: Weinheim, Germany, 2015; pp. 65-104 ISBN 9783527664610.

7. Harada, N.; Nakanishi, K. Exciton chirality method and its application to configurational and conformational studies of natural products. Acc. Chem. Res. 1972, 5, 257-263, doi:10.1021/ar50056a001.

8. A.S. Davydov The theory of molecular excitons. Sov. Phys. Usp. 1964, 7, 145-178.

9. Berova, N.; Di Bari, L.; Pescitelli, G. Application of electronic circular dichroism in configurational and conformational analysis of organic compounds. Chem. Soc. Rev. 2007, 36, 914-931, doi:10.1039/b515476f.

10. Alonso-Gómez, J. L.; Rivera-Fuentes, P.; Harada, N.; Berova, N.; Diederich, F. An enantiomerically pure alleno-acetylenic macrocycle: synthesis and rationalization of its outstanding chiroptical response. Angew. Chem. Int. Ed. 2009, 48, 5545-5548, doi:10.1002/anie.200901240.

11. Míguez-Lago, S.; Llamas-Saiz, A. L.; Magdalena Cid, M.; Alonso-Gómez, J. L. A Covalent Organic Helical Cage with Remarkable Chiroptical Amplification. Chem. - A Eur. J. 2015, 21, 18085-18088, doi:10.1002/chem.201503994.

12. Míguez-Lago, S.; Cid, M. M.; Alonso-Gómez, J. L. Covalent Organic Helical Cages as Sandwich Compound Containers. European J. Org. Chem. 2016, 2016, 5716-5721, doi:10.1002/ejoc.201600997.

13. Castro-Fernández, S.; Yang, R.; García, A. P.; Garzón, I. L.; Xu, H.; Petrovic, A. G.; Alonso-Gómez, J. L. Diverse Chiral Scaffolds from Diethynylspiranes: All-Carbon Double Helices and Flexible Shape-Persistent Macrocycles. Chem. - A Eur. J. 2017, 23, 11747-11751, doi:10.1002/chem.201702986.

14. Ozcelik, A.; Pereira-Cameselle, R.; von Weber, A.; Paszkiewicz, M.; Carlotti, M.; Paintner, T.; Zhang, L.; Lin, T.; Zhang, Y.-Q.; Barth, J. V.; van den Nobelen, T.; Chiechi, R. C.; Jakob, M.; Heiz, U.; Chiussi, S.; Kartouzian, A.; Klappenberger, F.; Alonso-Gómez, J. L. Device-Compatible Chiroptical Surfaces through Self-Assembly of Enantiopure Allenes. Langmuir 2018, 34, 4548-4553, doi:10.1021/acs.langmuir.8b00305.

15. Rivera-Fuentes, P.; Alonso-Gómez, J. L.; Petrovic, A. G.; Seiler, P.; Santoro, F.; Harada, N.; Berova, N.; Rzepa, H. S.; Diederich, F. Enantiomerically pure alleno-acetylenic macrocycles: synthesis, solid-state structures, chiroptical properties, and electron localization function analysis. Chem-Eur J 2010, 16, 
9796-9807, doi:10.1002/chem.201001087.

16. Castro-Fernández, S.; Cid, M. M.; López, C. S.; Alonso-Gómez, J. L. Opening Access to New Chiral Macrocycles: From Allenes to Spiranes. J. Phys. Chem. A 2015, 119, 1747-1753, doi:10.1021/jp508414r.

17. Levine, I. N. Quantum Chemistry; Englewood Cliffs: New Jersey, 1991;

18. Atkins, P. W. Physical Chemistry; fifth.; Oxford University Press, USA: Oxford, 1994;

19. Rosenfeld, L. Quantenmechanische Theorie der natürlichen optischen Aktivität von Flüssigkeiten und Gasen. Zeitschrift für Phys. 1929, 52, 161-174, doi:10.1007/BF01342393. 